\title{
Design and Build a Database Management System for Computer Science Department Students
}

Ryan Youssef Yaacoub

College of Computer Science and

Mathematics

University of Mosul, Iraq

\section{Received on: 19/10/2010}

Muhammad Hashem Sultan

Al-Nebras Secondary School

General Education Directorate /

Nineveh

Accepted on: 16/08/2011

\section{ABSTRACT}

Student's system has been analyzed and A database was designed according to their registration, study and lectures attending, with full documentation to their degrees, the information of undergraduate study in computer science department students University of Mosul are used as a case study. This research use two data models to represent the database first is the Entity-Relationship Model, second is the Relational Model, Microsoft Visual FoxPro 9.0 used to create the database which contain all the related tables, a numbers of important suitable windows and reports that matches the department's needs are created also.

Keywords: database, Entity-Relationship Model, Relational Model, Microsoft Visual FoxPro 9.0.

$$
\begin{aligned}
& \text { تصميم وبناء نظام إدارة قاعدة بيانات لطلبة قسم علوم الحاسوب } \\
& \text { محمد هاشم سلطان } \\
& \text { ريان يوسف يعقوب تصني } \\
& \text { ثانوية النبراس للبنين } \\
& \text { مديرية التربية العامة / نينوى لتارئ لانين } \\
& \text { تاريخ قبول البحث: 2011/08/16 } \\
& \text { كلية علوم الحاسوب والرياضيات } \\
& \text { جامعة الموصل } \\
& \text { تاريخ استلام البحث: 2010/10/19 }
\end{aligned}
$$

\section{الملخص}

تم في هذا البحث تحليل نظام الطلبة وتصميم نظام إدارة بياناتهم, من حيث تسجيل الطلبة ودوامهم وحضورهم للمحاضرات وأداءهم للامتحانات مع توثيق الدرجات, استخدمت البيانات الخاصة لطلبة الدراسات الأولية

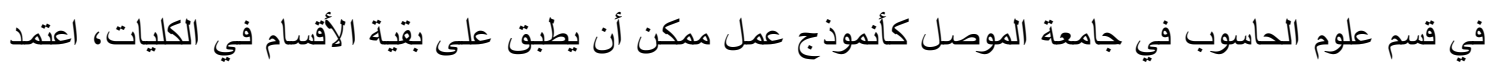
البحث في منهجيته على تحليل وتصميم النظام وذلك عن طريق تحديد التواجدات وتكوين نموذج التواجد - العلاقة (ER-Model ) البحث تم تكوين قاعدة البيانات التي تتضمن ربط عدد من الجداول ذات الصفات و الارتباطات (Relations) الناتجة من التحويل باستخدام تطبيق (Microsoft Visual FoxPro 9.0), تم إنشاء عدد من التقارير ذات الأهمية المناسبة واحتياجات الوحدات الإدارية والعلمية في القسم.

الكلمات المفتاحية: قاعدة بيانات، نموذج التواجد-العلاقة، النموذج العلائقي، مايكروسوف فيجول فولية فوكس برو 9.0. المقدمة

يعتبر موضوع بناء نظام إدارة قاعدة بيانات من المواضيع المهمة في مجال برمجيات الحاسوب حيث إن

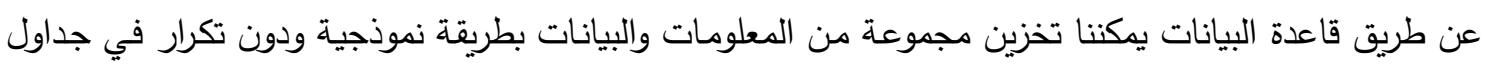

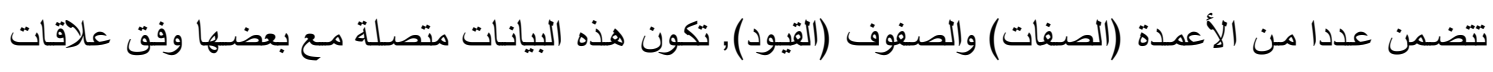

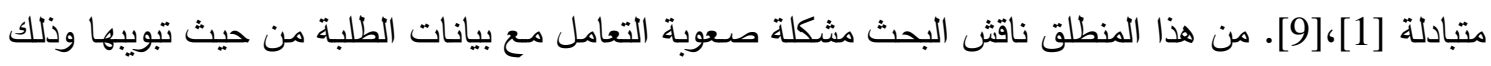

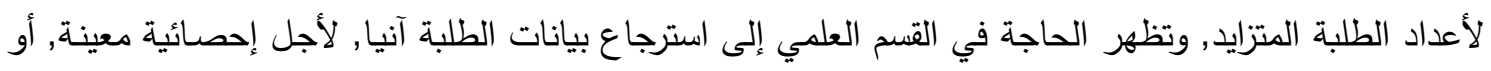


استعلام عن معلومات طالب معين, فبدلاً من الرجوع إلى السجلات والتي قد تكون أحيانا غير محدثة للوقت

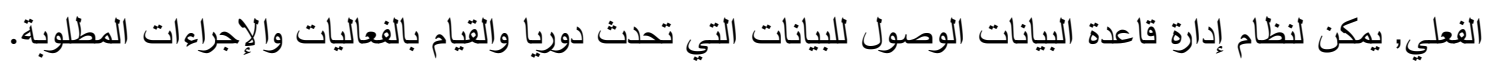
كما أن هنالك إحصائيات مطلوبة سواءً من مقرر القسم أو اللجنة الإمتحانية, مثلاً عدد الطلبة المستمرين بالدراسة, الطلبة الذين تجاوزت نسبة غيابهم الحد المسموح ,الطلبة الساكنين خارج مركز المحافظة, وطلبة الأقسام

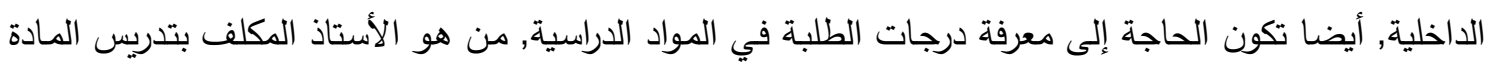
في السنة الدراسية الحالية أو لسنة سابقة وما هي المواد المشتركة بين الأساتذة.

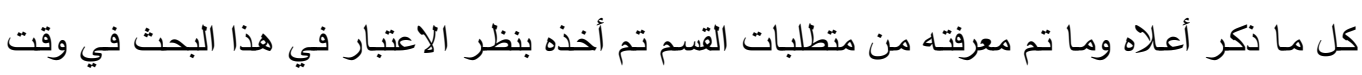
إعداده لتصميم وبناء نظام إدارة قاعدة البيانات لطلبة قسم علوم الحاسوب. تصميم وبناء قاعدة البيانات : إن عملية بناء قاعدة بيانات جيدة لا يأتي بتلك السهولة، إذ لابد من بذل جهد كبير للحصول على قاعدة

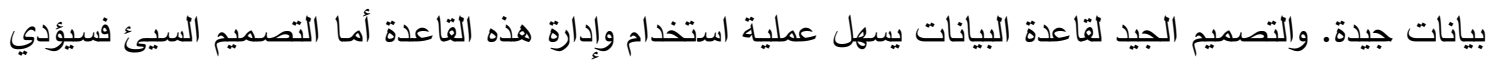
إلى تكرار البيانات Data Redundancy (وجود نفس البيانات في أكثر من مكان) [3] وبالتالي تصعب عملية

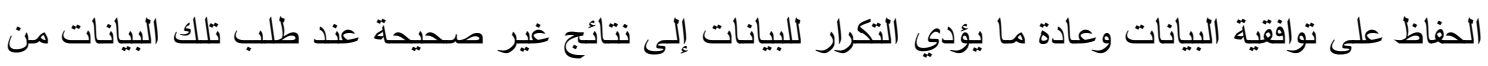
تلك القاعدة [5] وهذا بدوره يؤدي إلى أن أي قرارات إداريـة وكذلك أي تخطيط مستقبلي سيكون خاطئًا لاعتماده على معلومات غير صحيحة.

تم تصميم قاعدة البيانات ونظام إدارتها في هذا البحث حسب الخطوات التالية [1] [1] : أ. بناء النموذج التوضيحي (Conceptual Model) وتثمل هذه العملية عدة خطوات: تحليل البيانات ومتطلبات المستخدمين والإجراءات المطلوبة.

تعريف وتحديد التواجدات وخصائصها مع بعضها وكذلك وضعها في الصيغة المعيارية.

رسم المخطط التوضيحي وهو عبارة عن نموذج رسومي يوصف تواجدات النظام وعلاقتها مع بعضها. تعديل النموذج بحيث يثمل الإجراءات الرئيسية، وقواعد عمليات الإضافة والتعديل والحذف على على البيانات والتقارير ، والثاشات، ومقدار التثاركية وتوافقية البيانات.

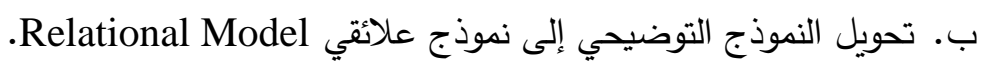

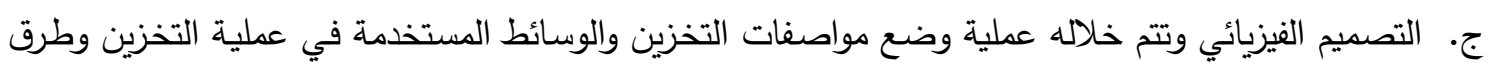

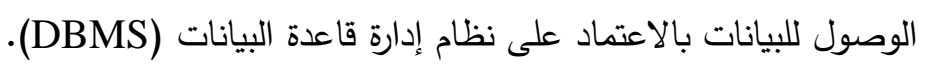

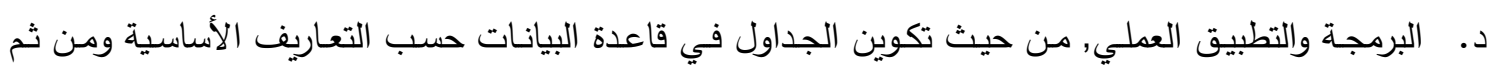
تكوين المشروع العملي لنظام إدارة قاعدة البيانات ( DBMS ) بجميع مكوناته, تكوين نوافذ الاختيار والإدخال وطباعة التقارير · تكونئ

Entity - Relationship Model أ. تكوين النموذج التوضيحي: نموذج التواجد- العلاقة إن هدف عملية التصميم هو الوصول إلى فهم صحيح للنظام للمساعدة في عملية تطوير هذا النظام، وهذا ليس بالأمر السهل إذ لابد من وجود مقياس صحيح للحكم على الفهم [4]. 


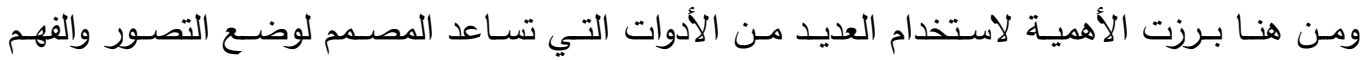
الصحيحين لعمل هذا النظام [3] [7]، ومن هذه الأدوات استخدام النماذج التوضيحية التي تصف لتف مكونات النظام وكيفية ارتباطها مع بعضها. تحتوي أية قاعدة البيانات على عناصر النماذج التالية[3], [4],[5]:1. (Entity Set) (

وتمثل المجموعة التي تتمي إليها مجموعة الكائنات (Objects) المتثابهة التي تكون محط الاهتمام في النظام وتمثل بجدول في قاعدة البيانات العلائقية. وعلينا أن نقوم بجمع وتسجيل البيانات عن هذا التواجد مثلاً الطالب، الأستاذ، المادة, التي تعد من التواجدات المهمة في نظام قاعدة بيانات الطلبة ويرمز لمجموعة التواجدات بمستطيل يحتوي على أسم التواجد.

2. (Attributes) (الصفات):

(إضـافة إلـى تعريفهـا السـابق) وهـي عبـارة عـن الخصـائص أو المحـدات المميـزة للتواجد، وبعبـارة

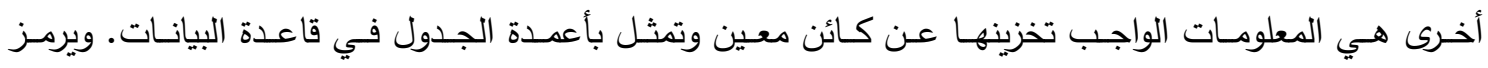

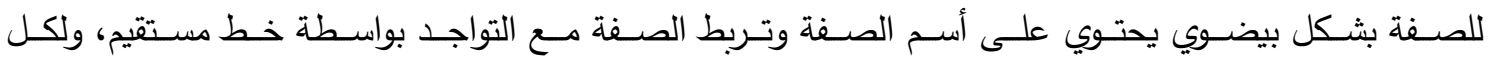
صفة لها مجال القيم(Domain).

3. العلاقات مابين التواجدات (Relationships among Entities):

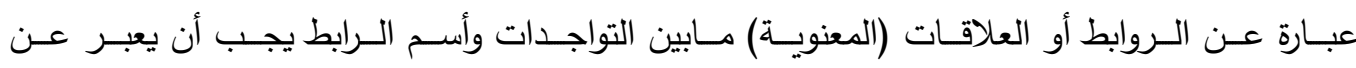

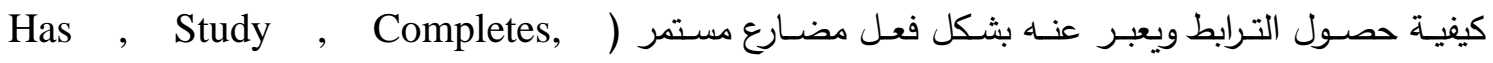
. (الخ). Belong To يرمـز لهـا بثـكل معـين يحتـوي علـى أســم الـرابط أو العلاقـة، وكـذلك لكـل علاقـة درجـة تثــاركية

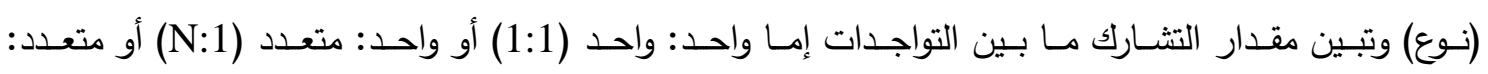

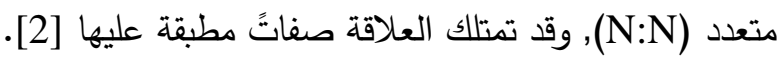

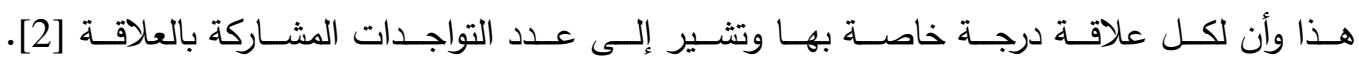

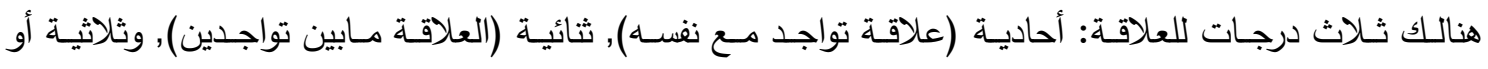
متعددة (العلاقة مابين أكثر من تواجدين) [4].

\section{[4],[2]: ER- Model التحليل النظري وتصميم نموذج التواجد - العلاقة}

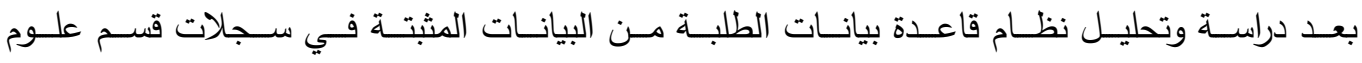

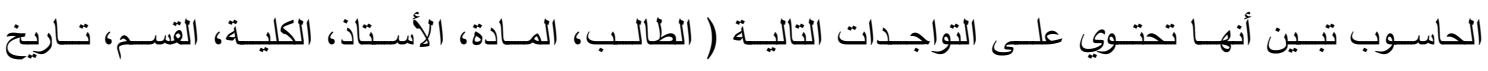

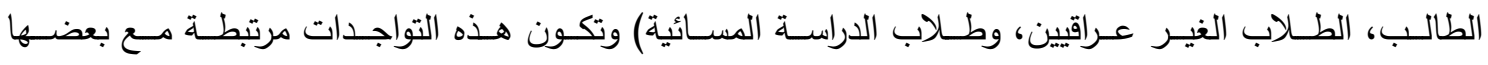

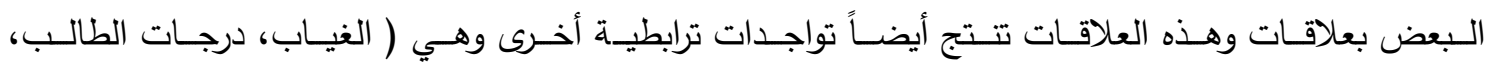
تأهيـل المـادة سـنوياً ), أمــا أنـواع الصـفات الموجـودة كانــت (الصـفات البسـيطة Simple Attributes

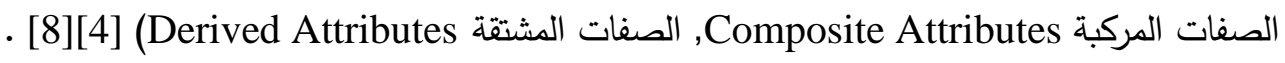


1. تواجد الطالب : Student Entity ويتضمن الصفات التالية:-

(رقم الطالب Student Number, أسم الطالب Student Name, تاريخ الولادة Date Of Birth, جنس الطالب Student Sex, جنسية الطالب Student Nationality, عنوان الطالب الثابت Sroven Address, عنوان الطالب الحالي Current Address, سنة القبول Accepting Year, سنة التخرج للطالب Student

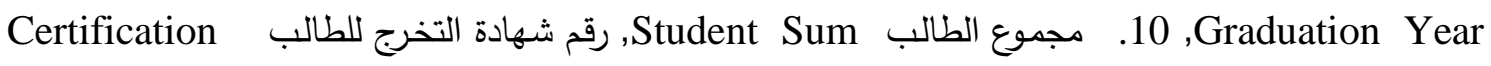
Student قسم الطالب, Student Graduation Year .(Department 2. تواجد الطالب المسائي Student Evening Entity ويتضمن الصفات التالية:(رقم الطالب المسائي Student Evening Number, رقم الفاتورة المدفوعة Bill Number). 3. تواجد الطالب الغير عراقي الجنسية The International Student Entity يتضمن الصفات التالية:(رقم الطالب الغير عراقي International Student Number, البلد Country). 4. تواجد المادة Course Entity ويتضمن الصفات التالية:-

(رقم المـادة Course Number, أسم المـادة باللغـة العربيـة Arabic Course Name, أسم المـادة باللغـة

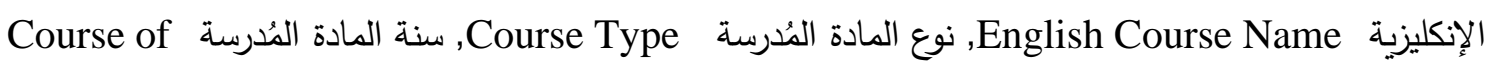
Year 5. تواجد أستاذ المادة Course Professor Entity ويتضمن الصفات التالية:(رقم الأستاذ Professor Number, أسم الأستاذ Professor Name, الثهادة الحاصل عليها الأستاذ Certificate of Professor للشهادة The Certificate Giver, رقم غرفة الأستاذ Professor Room Number). 6. تواجد الكلية College Entity ويتضمن الصفات التالية:(رقم الكلية College Number, أسم الكلية College Name, أسم العيد Dean Name, سنة تأسيس الكلية College Establish Year). 7. تواجد القسم Department Entity ويتضمن الصفات التالية:(رقم القسم Department Number, أسم القسم Department Name, أسم رئيس القسم Department,

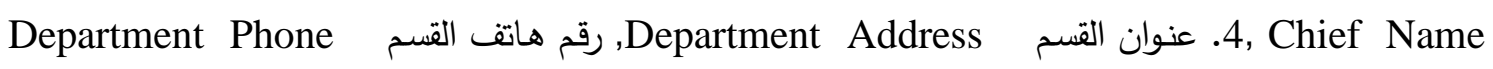
(Number 8. تواجد السنة الاراسية للطالب Scholastic Year for Student Entity ويتضمن الصفات التالية:- 
(السنة Year, حالة الطالب Student State, ملحق الحالة State Appendix, المرحلة The Stage, (The Class الثعبة

Relationships ثانيا: العلاقات

العلاقة رقم (1): (STUDY)

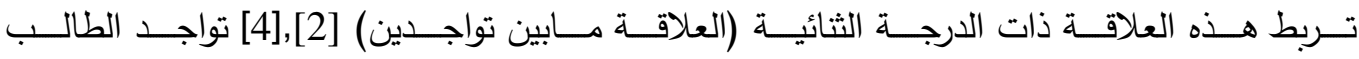

[4] (many to optional many mandatory) وهي من نوع (COURSE) مع تواجد المادة (STUDENT) بحيث انه يمكن لطالب أو عدد من الطلاب أن يدرسون مادة أو عدد من المواد، وتكون (mandatory) إجبارية

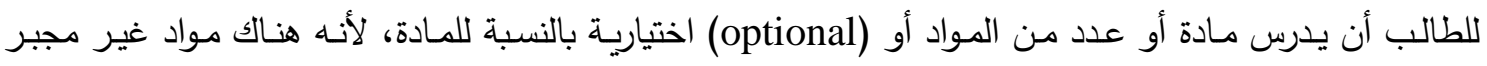

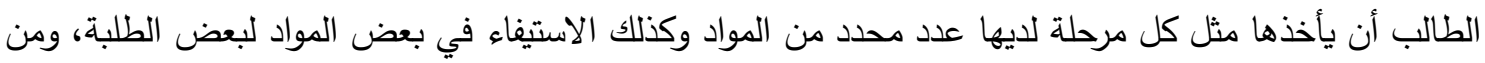
خلال هذه العلاقة نستطيع أن نعرف درجات الطلاب. تمتلك هذه العلاقة الصفات (Attributes) التالية: (درجة الطالب للفصل الأول 1 Course Degree, درجة الطالب في نصف السنة Course Degree 2 (A) درجة الطالب للفصل الثاني Course Degree 3 درجة الطالب النهائية للدور الأول: دinal Exame1 Degree, درجة الطالب النهائية للدور الثاني Final Exame2 Degree, درجة الطالب النهائية Final Degree).

\section{العلاقة رقم (2) (علاقة Supertype/Subtype) [4],[8]}

تربط العلاقـة الثانيـة تواجد الطالب (STUDENT) مـع تواجد الطالب المسـئي ( ) EVENING

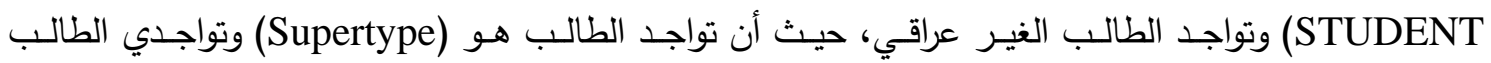
المسائي والغير عراقي هما (Subtypes) اللذان يعتمدان على تواجد رئيسي (أعلى) وهو تواجد الطالب, هذه

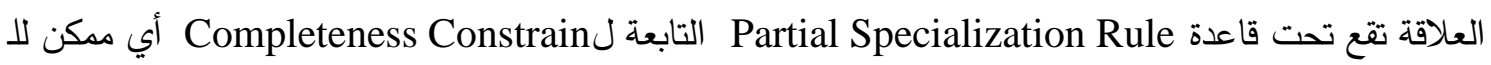
أن ائد للا Supertype أن لاعمل بياناته في أي Subtype, حيث مثلت بشكل خط مفرد مرسوم من

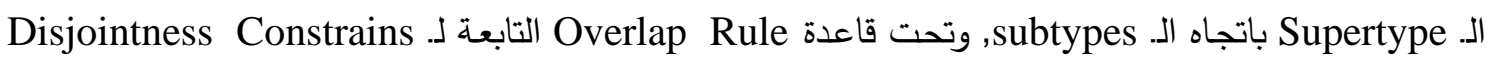

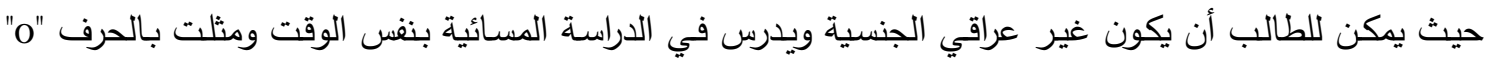

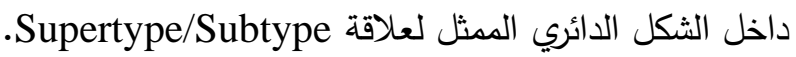
(STUDY IN) (3) العلاقة رقم تربط هذه العلاقة تواجد الطالب (STUDENT) مع تواجد القسم (DEPARTMENT) بعلاقة ثائية من نوع (Optional One to Mandatory One) (الطالب يدرس في القسم) تكون اختيارية للطالب بأن ينتمي إلى قسم معين ولكن إجبارية من جهة القسم لأنه يجب أن يحتوي القسم على طلاب. (4AS_HISTORY) (4) العلاقة رقم (H) تربط هذه العلاقة تواجد الطالب (STUDENT) مع تواجد سنة الدراسة (STUDY YEAR) بعلاقة والتي تعني أن الطالب يمتلك تاريخ لحالات قد مر بها من (Mandatory One to Mandatory Many) (أي السيرة الدراسية للطالب) حيث النجاح أو الرسوب أو ترقين القيد. 
(COURSE QUALIFYING) (5) العلاقة رقم (5)

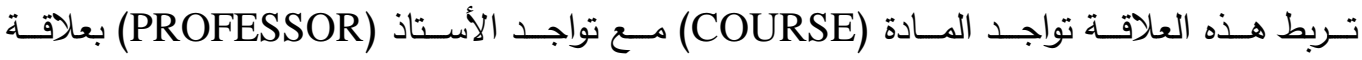

ثنائيسة مـن نـوع (Optional Many to Mandatory Many) والتـي تعنـي تأهيـل المـادة مـن قبـل

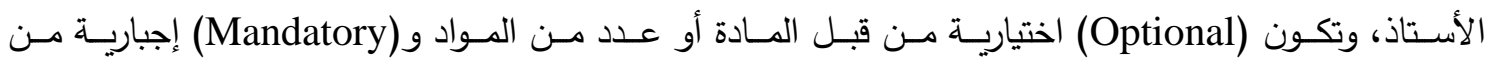

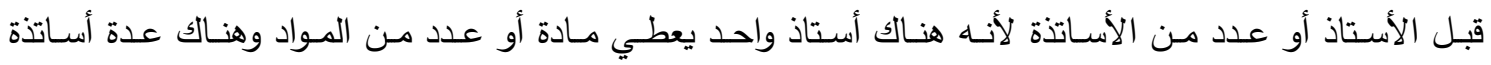

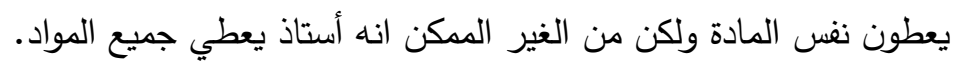
تمتلك هذه العلاقة الصفات (Attributes) التالية: ( تاريخ بداية تدريس المادة Beginning Course Date, تاريخ نهاية تدريس المادة (Anding Course Date).

(GIVE ABSENCE) (6) العلاقة رقم

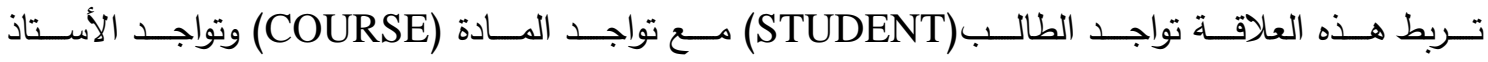

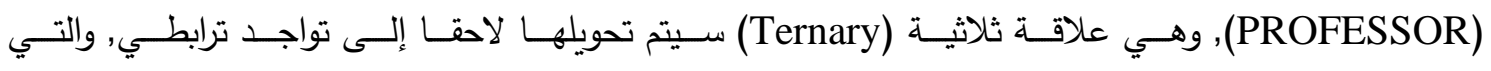
تفيدنا في أعطـاء غيابـات الطالب, حيث يمكن بواسطة التواجد الترابطي معرفـة غيابـات الطالب في المـادة التي يُدرسها أُستاذ معين وبالتاريخ المعين. تُعاب. تمتلك هذه العلاقة صفة التاريخ: Date (Belong to) (7) العلاقة رقم تربط هذه العلاقة تواجد الكلية (COLLEGE) مع تواجد القسم (DEPARTMENT) بعلاقة ثنائية من نوع والعلاقة تعني انه قسم واحد أو عدد من الأقسام ينتمي إلى كلية واحدة. وليس إلى عدة من (One to Many)

ب. تحويل نموذج التواجد-العلاقة إلى النموذج العلانقي [1],[4],]: يعد النموذج العلائقي احد أهم النماذج المنطقية لتمثيل قواعد البيانات, تم اقتراح هذا النموذج في العام 1970 من قبل العالم Edgar Frank Codd, مع نظريات الجبر البولي Boolean Algebra, حاليا يعد النموذج العلائقي التقنية المهيمنة على تصميم قواعد البيانات المنطقية, يهتم هذا النموذج بتكوين الجداول (Relation) والعلاقات مابين تلك الجداول باستخدام المفاتيح (الفهارس) الأساسية والأجنبية. تعرف الرابطة (Relation) بأنها عبارة عن جدول ثنائي الأبعاد يحمل اسماً وحيدا في قاعدة البيانات, يتضمن

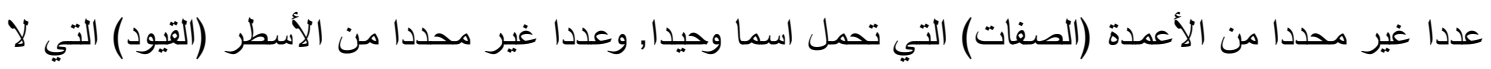
تحمل اسما وغير قابلة للتكرار • ويجب أن تمتلك مفتاحاً أساسيا (بسيطا أو مركباً).

أنواع المفاتيح في قاعدة البيانات : 1. المفتاح الأساسي (Primary Key): هو عبارة عن صفة أو مجموعة من الصفات تميز بصورة وحيدة سطراً في الجدول (Relation). (2) (1) 
2. المفتاح الأجنبي (Foreign Key): هو عبارة عن صفة (أو مجموعة من الصفات) في جدول تخدم كونها مفتاحا أساسيا في جدول آخر في نفس قاعدة البيانات.

(في التمثيل البياني للنموذج العلائقي يتم التعرف على المفاتيح الأساسية والأجنبية بان يتم مد خط متصل

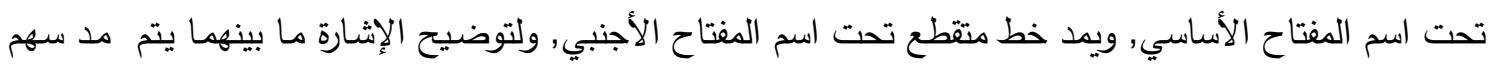

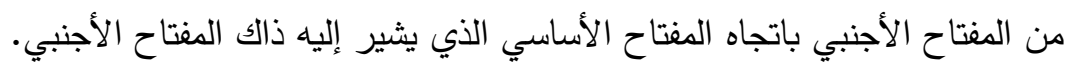
لتحويل التصميم إلى قاعدة بيانات لابد في البدايـة من تحويل نموذج التواجدات والعلاقات إلى نموذج علائقي (Relational Model) حتى نسهل عملية تتفيذ هذا النموذج في قاعدة بيانات (إنشاء الجداول) وسنقوم أولاً بدراسة كيفية تحول نموذج التوضيحي (نموذج التواجدات والعلاقات) إلى نموذج علائقي.

أ. لكل تواجد (Entity) في النموذج نقوم بإنشاء علاقة (Relation) بحيث تحتوي العلاقة على جميع الصفات

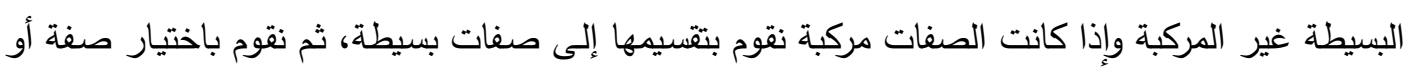
أكثر لتشكل المفتاح الرئيس للعلاقة.

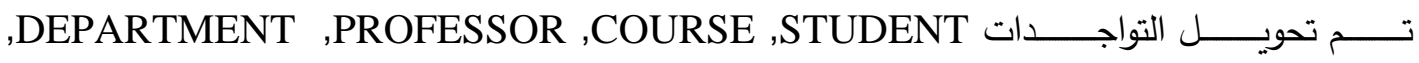
STUDY YEAR ,INTERNATIONAL STUDENT,EVENING STD ,COLLEGE (Relations)

STUDENT

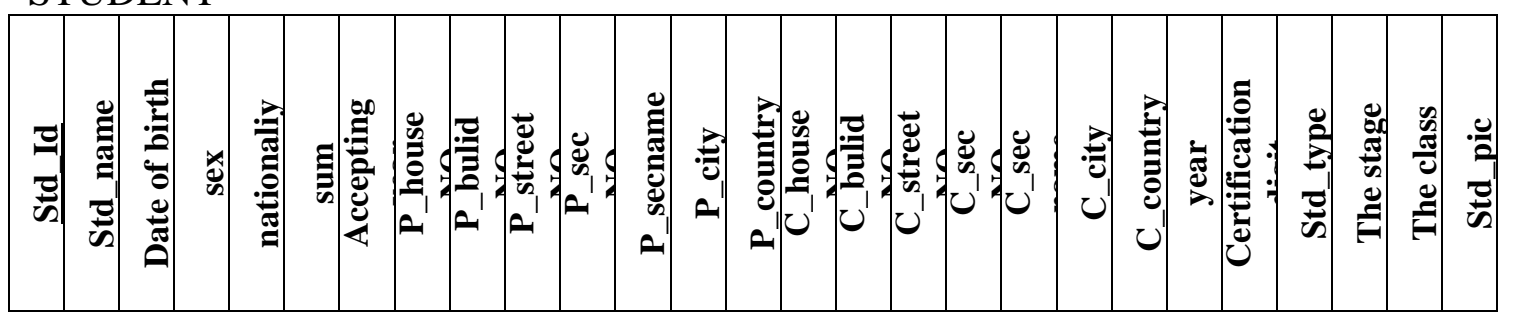

COURSE

\begin{tabular}{c|c|c|c|c|c|}
\hline Cousrse_id & Arabic_course_Name & English_Course_Name & Course_Year & Course_Type & No_Of_Units \\
\hline
\end{tabular}

PROFESSOR

\begin{tabular}{|l|l|l|l|l|l} 
Prof_id & Prof_name & certificate & Certificate_Year & The_Certificate_Giver & Prof_Room_No \\
\hline
\end{tabular}

DEPARTMENT

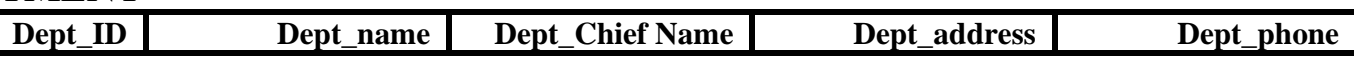

COLLEGE

\begin{tabular}{l|l|l|l} 
Col_ID & Col_Name & Col_Dean_name & Establish_year
\end{tabular}

EVENING STD

EStd_ID $\quad$ Bill_NO

INTERNATIONAL STUDENT

\begin{tabular}{l|l} 
NStd_ID & Country \\
\hline
\end{tabular} 
STUDY YEAR

C Year

\begin{tabular}{l|l|l|l} 
State & Appendix & The_stage & The_class \\
\hline
\end{tabular}

الثكل (1). الجداول (Relations) الناتجة من تحويل التواجدات:

(STUDENT , COURSE,PROFESSOR ,DEPARTMENT , COLLEGE, EVENING STD ,

INTERNATIONAL STUDENT,STUDY YEAR)

ب. تحويل العلاقات حسب التثاركية :

كما مر علينا سابقاً فهناك ثلاثة أنواع من التثاركية(نوع العلاقة) علاقة واحد: واحد (1:1) وعلاقة واحد:

متعدد (N:1) وعلاقة متعدد: متعدد (M:N).

يكون التحويل حسب الخطوات التالية:

One to One : 1 1:1:1) علاقة واحد: واحد

لكل علاقة واحد: واحد (1:1) نقوم باختيار أحد التواجدين في الجهة الاختيارية من المشاركة ليحتوي على

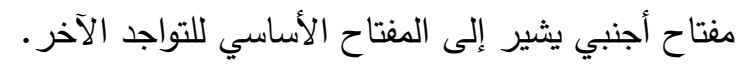

One to Many :(N:1) علاقة واحد: متعدد

لكل علاقة واحد: متعدد (N:1) نقوم بإضافة حقل جديد ليكون مفتاحاً أجنبياً في جانب المتعدد (N) ليشير

إلى المفتاح الرئيسي في جانب الواحد (1).

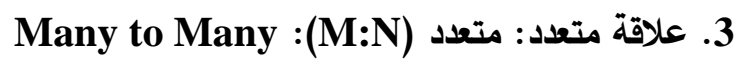

لكل علاقة متعدد: متعدد (N:N) نقوم بإنثاء علاقة جديدة يكون المفتاح الرئيسي لها عبارة عن دمـج المفاتيح في طرفي العلاقة وإضافة أي صفات موضوعة على العلاقة.

4. تحويل العلاقة بين الأنواع الفرعية (Subtype) والأنواع العليا (Super Type):

وذلك عن طريق وضع المفتاح الرئيس في النوع الفرعي ليكون مفتاحاً رئيسياً لهذا الجدول وفي نفس الوقت

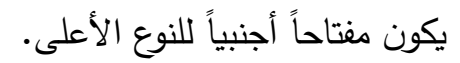

فيما يلي يتم تحويل نموذج العلاقات التي ربطت بين الجداول والتي ذكرت سابقاً وبالاعتماد على الشروط الأنفة الذكر وربطها مع بعضها البعض:

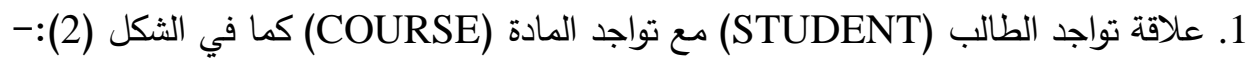

STUDENT

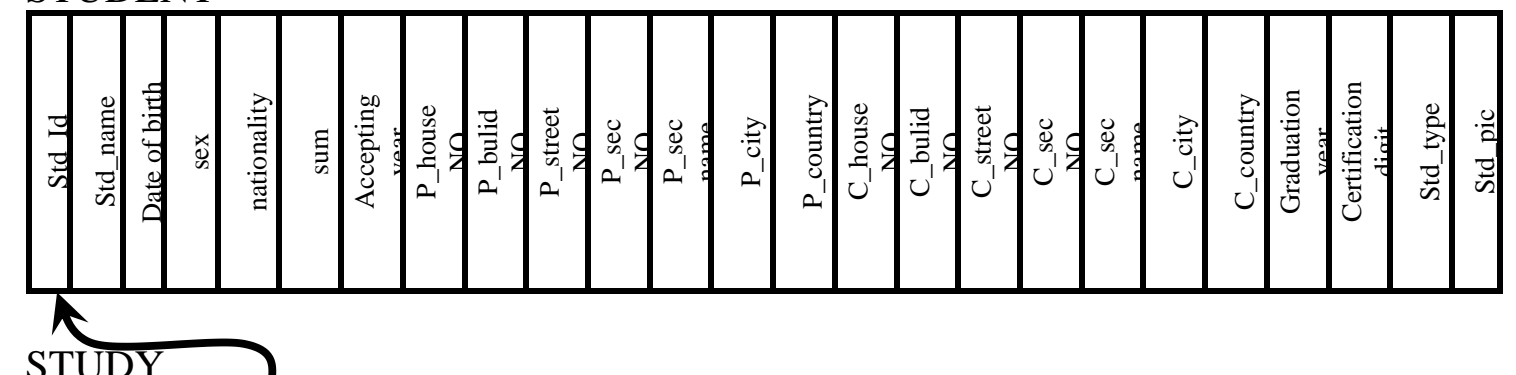

\begin{tabular}{|c|c|c|c|c|l|l|l|l|}
\hline$\underline{\text { Std id }}$ & Course id & C_degree1 & C_degree2 & C_degree3 & Endeavoring & $\begin{array}{c}\text { F_degree } \\
\text { Ex1 }\end{array}$ & $\begin{array}{c}\text { F_degree } \\
\text { Ex2 }\end{array}$ & F_degree \\
\hline
\end{tabular}

COURSE

Course id

Arabic_course_name

English_course_name

Course_Year

Course_Type

No_Of_Units

الثكل (2). الجداول (Relations) الناتجة من تحويل علاقة تواجد الطالب (STUDENT) مع تواجد المادة

(COURSE) 
2. علاقة تواجد الطالب ( STUDENT ) مع توجد المادة ( COURSE ) وتواجد الأستاذ (PROFESSOR) كما في الثكل (3):-

STUDENT

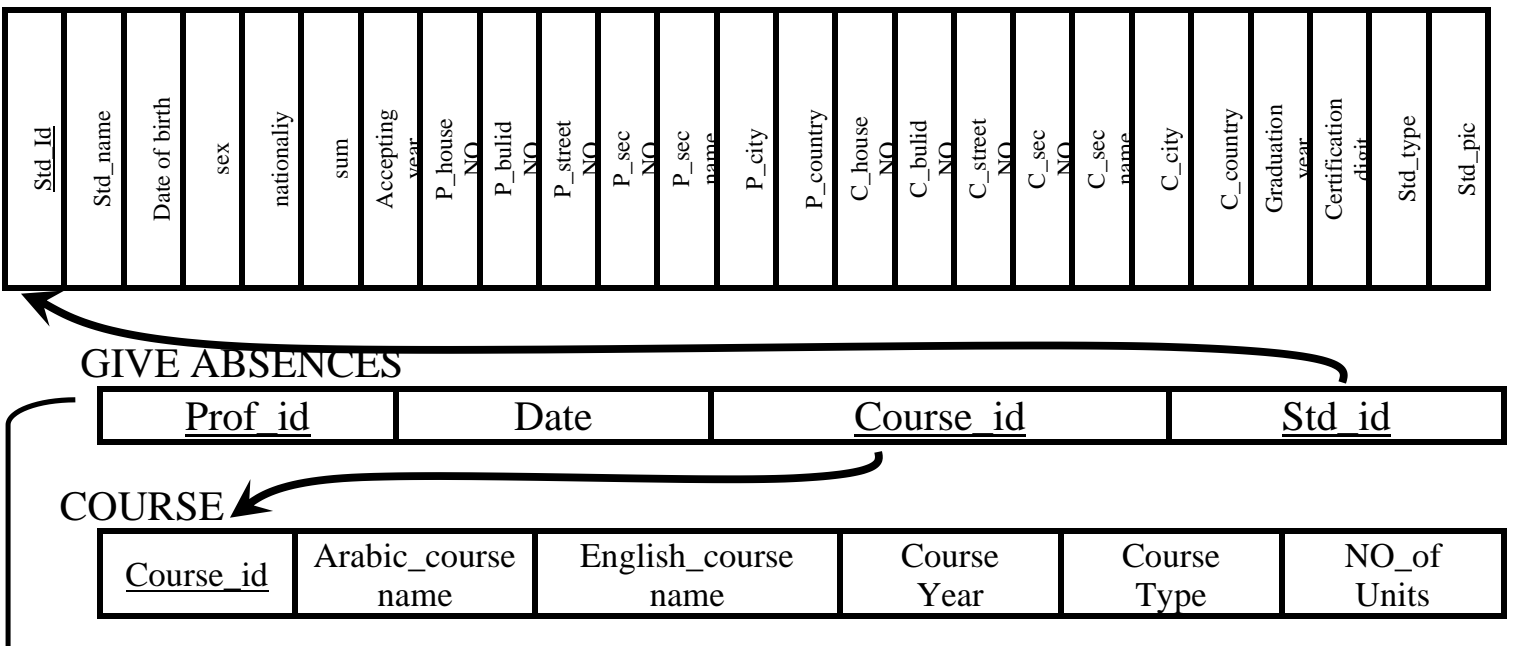

PROFESSOR

\begin{tabular}{|l|l|l|l|l|l|}
\hline Prof_id & Prof_name & certificate & Certificate_year & The_certificate_Giver & Prof_Room_No \\
\hline
\end{tabular}

الثكل (3). الجداول (Relations) الناتجة من تحويل علاقة تواجد الطالب (STUDENT) مع تواجد المادة

(PROFESSOR ) وتواجد الأستاذ (COURSE)

3. علاقـة Supertype/Subtype التـي تـربط تواجــ الطالب (STUDENT) مـع تواجـــ الطالـب المســئي

(EVENING STD)

INTERNATIONAL STUDENT

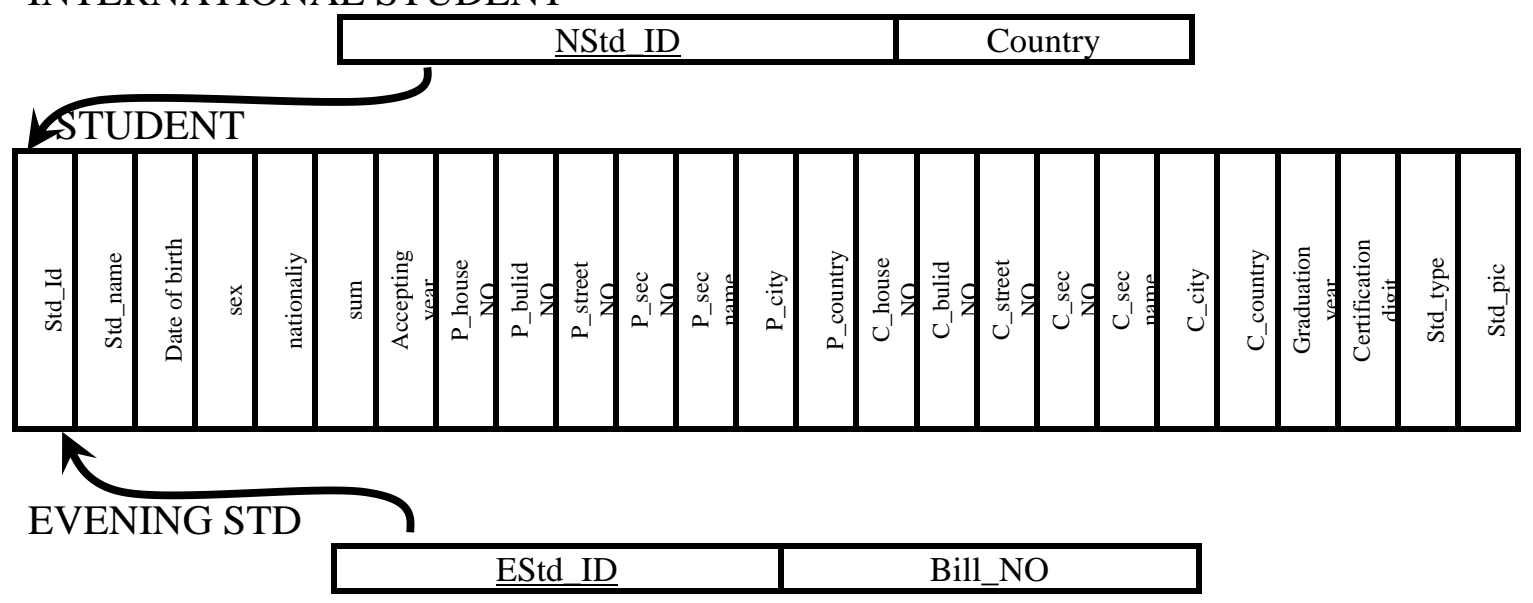

الثكل (4). الجداول (Relations) الناتجة من تحويل علاقة Supertype/Subtype التي تربط تواجد الطالب

INTERNATIONAL ) مع تواجد الطالب المسائي (EVENING STD) والطالب الغير عراقي (STDENT)

(STUDENT

4. علاقة تواجد الطالب (STUDENT) مع تواجد القسم (DEPARTMENT) كما في الشكل(5):- 


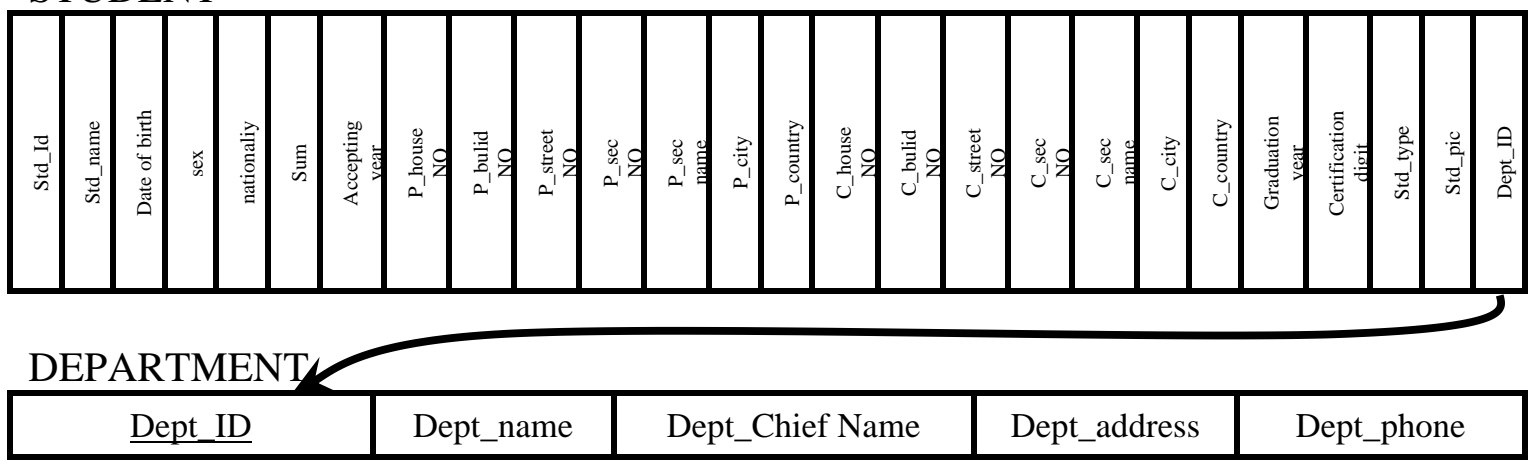

الثكل (5). الجداول (Relations) الناتجة من تحويل علاقة تواجد الطالب (STUDENT) مع تواجد القسم

(DEPARTMENT)

5. علاقة تواجد الطالب (STUDENT) مع تواجد السنة الدراسية (STUDY YEAR) كما في الثكل (6):STUDENT

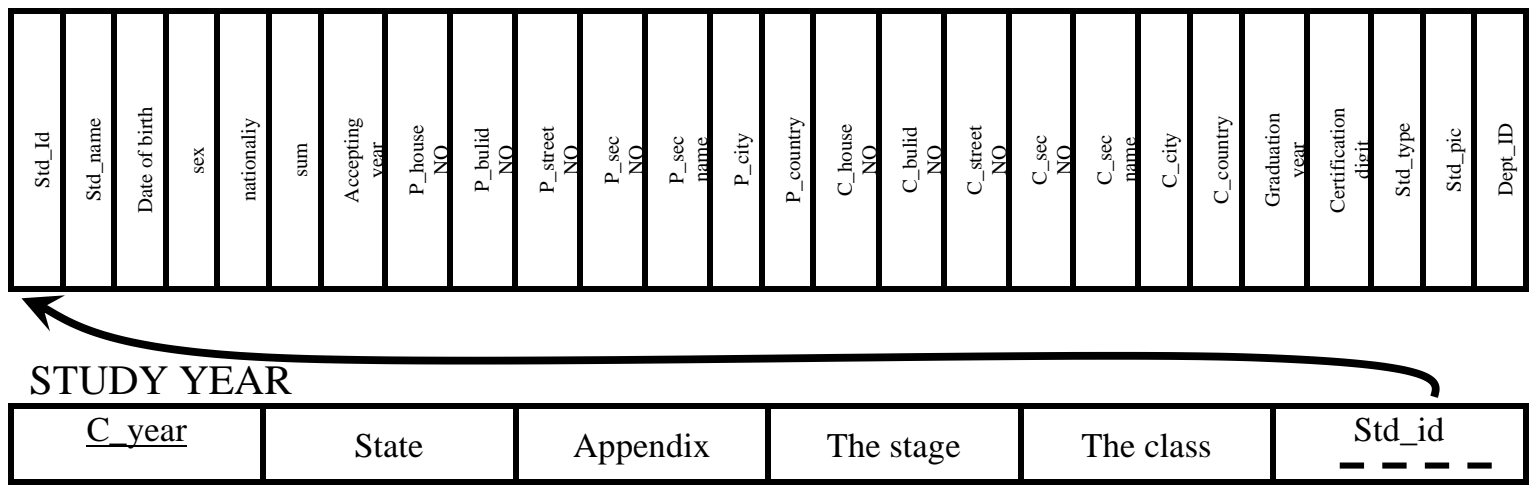

الثكل (6) (Relations) الناتجة من تحويل علاقة تواجد الطالب (STUDENT) مع تواجد السنة الدراسية

(STUDY YEAR)

6. علاقة تواجد المادة (COURSE) مع تواجد الأستاذ (PROFESSOR) كما في الثكل (7):COURSE

\begin{tabular}{|c|l|l|l|l|l|}
\hline Course_id & Arabic_course_name & English_course_name & Course_year & Course_type & No_Of_Units \\
\hline
\end{tabular}

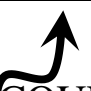

COURSE QUALIFYING

\begin{tabular}{l}
\hline \multicolumn{2}{|c|}{ Course_id } \\
\begin{tabular}{|c|c|c|c|c|c|}
\hline \multicolumn{2}{|c|}{ Prof_id } & Beginning course date & Ending course date \\
\hline Prof_id & Prof_name & certificate & Certificate_year & The certificate_Giver & Prof_room_NO \\
\hline
\end{tabular}
\end{tabular}

الثكل (7). الجداول (Relations) الناتجة من تحويل علاقة تواجد المادة (COURSE) مع تواجد الأستاذ

(PROFESSOR)

7. علاقة تواجد القسم (COLLEGE) (DEPARTMENT) مع تواجد الكلية في الثكل (8):- 


\section{DEPARTMENT}

\begin{tabular}{|c|c|c|c|c|c|}
\hline$\underline{\text { Dept ID }}$ & Dept_name & Dept_Chief Name & Dept_address & Dept_phone & Col_id \\
\hline COLLEGE & Col_Name & Col_Dean_name & Establish_year \\
\hline
\end{tabular}

الثكل (8). الجداول (Relations) الناتجة من تحويل علاقة تواجد المادة (COURSE) مع تواجد الأستاذ

(PROFESSOR)

\section{التقصي عن كون الجداول (Relations) في الصيغة الطبيعية:}

أجريت عملية التطبيع (Normalization) على الـ Relations أعلاه وكانت جميعها ضمن الصيغة

الطبيعية الثالثة 3NF لعدم وجود أية صفة متعددة, وجميع الصفات الغير مفتاحية تعتمد وظيفياً بصورة كلية على

المفتاح الأساسي أو المجموعة المؤلفة له, ولم يكن هنالك أي اعتماد وظيفي مابين الصفات الغير مفتاحية [3].

$$
\text { ج • التصميم الفعلي [2],[4]: }
$$

خلال التصديم الفعلي يتم تحديد خصائص الصفات لكل جدول (Relation) ومن خلال الواقع العملي المطلوب والذي تم معرفته خلال تحليل قاعدة البيانات تم تخصيص نوع البيانات والحجم لكل صفة (عمود) لكل

$$
\text { جدول وحسب جداول وصف النطاق (1)-(11) التالية : }
$$

\begin{tabular}{|c|c|c|}
\hline Attribute (الصفة) & Data Type (النوع) & Description (الوصف) \\
\hline Std_ID & Integer (4) & رقم الطالب (مفتاح أساسي) \\
\hline Std_name & Character (40) & أسم الطالب الثلاثي \\
\hline Date_of_brith & Date & تاريخ الولادة \\
\hline Sex & Character (1) & الجنس (ذكر أو أنثى) تكون القيمة حرف واحد إما M (Male) أو Female) \\
\hline Nationality & Character (15) & الجنسية \\
\hline Sum & Float $(6,2)$ & مجموع الطالب للاراسة الثانوية \\
\hline Accepting_year & Numeric (10) & سنة القبول في الكلية \\
\hline P_house_no & Numeric (3) & رقم البيت المثبت \\
\hline P_bulid_no & Numeric (3) & رقم البناية المثبت \\
\hline P_street_no & Numeric (3) & رقم الثارع المثبت \\
\hline P_sec_no & Numeric (4) & رقم القطاع المثبت \\
\hline P_sec_name & Character (15) & أسم القطاع المثبت \\
\hline P_city_name & Character (12) & أسم المدينة المثبت \\
\hline P_country_name & Character (20) & أسم البلد المثبت \\
\hline C_house_no & Numeric (3) & رقم البيت الحالي \\
\hline C_bulid_no & Numeric (3) & رقم البناية الحالي \\
\hline C_street_no & Numeric (3) & رقم الشارع الحالي \\
\hline C_sec_no & Numeric (4) & رقم القطاع الحالي \\
\hline C_sec_name & Character (15) & أسم القطاع الحالي \\
\hline C_city_name & Character (12) & أسم المدينة الحالي \\
\hline C_country_name & Character (20) & أسم البلد الحالي \\
\hline
\end{tabular}

الجدول (1). وصف النطاق لجدول Student 


\begin{tabular}{|c|c|c|}
\hline Graduation_year & Numeric (9) & سنة التخرج \\
\hline Certificate_digit & Numeric (5) & رقم شهادة التخرج \\
\hline Std_type & Logical (1) & نوع الدراسة ( T T ) : صباحي ( F ) : مسائي \\
\hline Std_pic & General(4) & صورة الطالب \\
\hline
\end{tabular}

\begin{tabular}{|c|c|c|}
\hline Attribute (الصفة)) & Data Type (النوع) & Description (الوصف) \\
\hline Prof_ID & Integer (4) & رقم الأستاذ(مفتاح أساسي) \\
\hline Prof_name & Character (40) & أسم الأستاذ الثلاثي \\
\hline Certificate & Character (10) & الثهادة \\
\hline Certificate_giver & Character (40) & الجهة المانحة للشهادة \\
\hline Certificate_year & Numeric (10) & سنة منح الشهادة \\
\hline Prof_room_no & Numeric (4) & رقم غرفة الأستاذ \\
\hline
\end{tabular}

الجدول (3). وصف النطاق لجدول Course

\begin{tabular}{|c|c|c|}
\hline Attribute (الصفةة) & Data Type (النوع) & Description (الوصف) \\
\hline Course_ID & Character (2) & رمز المادة (مفتاح أساسي) \\
\hline Arabic_course_name & Character (20) & اسم المادة باللغة العربية \\
\hline English_course_name & Character (20) & اسم المادة باللغة الانجليزية \\
\hline Course_year & Numeric (10) & 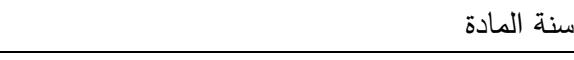 \\
\hline Course_type & Character (1) & نوع المادة (نظري (T) )، عملي (P)، مدموج (M) ) \\
\hline No_of_units & Numeric (2) & 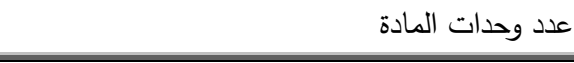 \\
\hline
\end{tabular}

\section{الجدول (4). وصف النطاق لجدول College}

\begin{tabular}{|c|c|c|}
\hline Attribute (الصفة)) & Data Type (النوع) & Description (الوصف) \\
\hline Col_ID & Character (4) & رمز الكلية (مفتاح أساسي) \\
\hline Col_name & Character (50) & 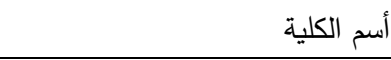 \\
\hline Dean_name & Character (50) & أسم العميد \\
\hline Establish_year & Numeric (10) & سنة تأسيس الكلية \\
\hline
\end{tabular}

Course qualifying الجدول (5). وصف النطاق لجدول

\begin{tabular}{|c|c|c|c|}
\hline Attribute (الصفة) & Data Type (النوع) & \multicolumn{2}{|c|}{ Description (الوصف) } \\
\hline Prof_ID & Integer (4) & \multirow[b]{2}{*}{ (مفتاح أساسي) } & رقم الأستاذ \\
\hline Course_ID & Character (2) & & رمز المادة \\
\hline Course_year & Character (10) & & سنة المادة \\
\hline Beging_course_date & Date & & تاريخ بداية الفصل \\
\hline Ending_course_date & Date & & تاريخ نهاية الفصل \\
\hline
\end{tabular}


الجدول (6). وصف النطاق لجدول Department

\begin{tabular}{|c|c|c|}
\hline Attribute (الصفة) & Data Type (النوع) & Description (الوصف) \\
\hline Dept_ID & Character (2) & رمز القسم (مفتاح أساسي) \\
\hline Dept_name & Character (40) & أسم القسم ـ ـ \\
\hline Head_Of_Dept_Name & Character (50) & أسم رئيس القسم \\
\hline Dept_address & Character (40) & عنون القسم \\
\hline Dept_phone & Numeric (20) & رقم هاتف القسم \\
\hline Col_ID & Character (4) & رمز الكلية \\
\hline
\end{tabular}

الجدول (7). وصف النطاق لجدول Evening student

\begin{tabular}{|l|l|r|}
\hline \multicolumn{1}{|c|}{ Attribute (الصفة) } & \multicolumn{1}{|c|}{ Data Type (النوصف) } \\
\hline Estd_ID & Integer (4) & Description \\
\hline Bill_no & Numeric (7) & \\
\hline
\end{tabular}

الجدول (8). وصف النطاق لجدول Gives absence

\begin{tabular}{|c|c|c|c|}
\hline Attribute (الصفة)) & Data Type (النوع) & \multicolumn{2}{|c|}{ Description (الوصف) } \\
\hline Std_ID & Integer (4) & \multirow[b]{2}{*}{ (مفتاح أساسي) } & رقم الطالب \\
\hline Corse_ID & Character (2) & & رقم المادة \\
\hline Course_year & Numeric (9) & & السنة \\
\hline Prof_ID & Character (8) & & رقم الأستاذ \\
\hline Date & Date & & التاريخ \\
\hline
\end{tabular}

Non_Iraqi الجدول (9). وصف النطاق لجدول

\begin{tabular}{|l|l|r|}
\hline \multicolumn{1}{|c|}{ Attribute (الصفة) } & \multicolumn{1}{|c|}{ Data Type (النوصف) } \\
\hline Std_ID & Integer (4) & Description \\
\hline Country & Character (20) & (البلد الطالب (مفتاح أساسي) \\
\hline
\end{tabular}

الجدول (10). وصف النطاق لجدول Study

\begin{tabular}{|c|c|c|c|}
\hline Attribute (الصفة) & Data Type (النوع) & \multicolumn{2}{|c|}{ Description (الوصف) } \\
\hline Std_ID & Ineger (4) & \multirow[b]{2}{*}{ (مفتاح أساسي) } & رقم الطالب \\
\hline Course_ID & Character (2) & & رقم المادة \\
\hline Course_year & Numeric (9) & & السنة - ال \\
\hline C_degree 1 & Float $(4,2)$ & & درجة الفصل الأول \\
\hline C_degree2 & Float $(4,2)$ & & درجة الفصل الثاني \\
\hline C_degree3 & Float $(4,2)$ & & درجة نصف السنة \\
\hline Endeavoring & Float $(4,2)$ & & السعي السنوي \\
\hline F_ex1_degree & Float $(4,2)$ & الأول & درجة امتحان الدور \\
\hline F_ex2_degree & Float $(4,2)$ & الثاني & درجة امتحان الدور \\
\hline F_degree & Float $(5,2)$ & & الدرجة النهائية \\
\hline
\end{tabular}


Study Year الجدول (11). وصف النطاق لجدول

\begin{tabular}{|c|c|c|}
\hline Attribute (الصفة) & Data Type (النوع) & Description (الوصف) \\
\hline Course_year & Numeric (9) & السنة (مفتاح أساسي) \\
\hline State & Character (1) & 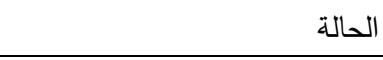 \\
\hline Appendix & Character (40) & توضيح للحالة \\
\hline Std_ID & Integer & رقم الطالب \\
\hline
\end{tabular}

د. البرمجة والتطبيق العملي (تكوين قاعدة البيانات والبرنامج التطبيقي لإدارتها): باستخدام تطبيق Microsoft Visual FoxPro 9.0 تم تكوين قاعدة البيانات للطلبة تحت اسم حسب تعريفات الجداول(1)- (11) السابقة وظهرت كما في الثكل (9) لم تكن هنالك أية عملية Denormalization [4] لان الجداول مصاغة بصورة جيدة وتم التعريف للحقول كليا في ملفات مستقلة, مع مراعاة ضوابط التكامل لكل حقل ذي إدخالات استثنائية ,استخدمت خواص البرمجة الثيئية في برمجة الواجهات,ولم يستخدم الـ Wisual FoxPro في أي جزء من البرنامج, وظفت Embedded SQL الخاصة بتطبيق لأجل إنشاء الاستعلامات والتقارير , تم تكوين ملف المشروع (FoxPro Project) باللغة سابقة الذكر , إما برنامج التتصيب (Setup) فقد تم تكوينه باستخدام 5.0 Install Shield Express الذي يقوم بدعم البرنامج التنفيذي كي بـاه يكون بالإمكان تشغيله على أية حاسبة بدون الحاجة لوجود اللغة التي بردج بها في الحاسبة المنشودة للتصيب.

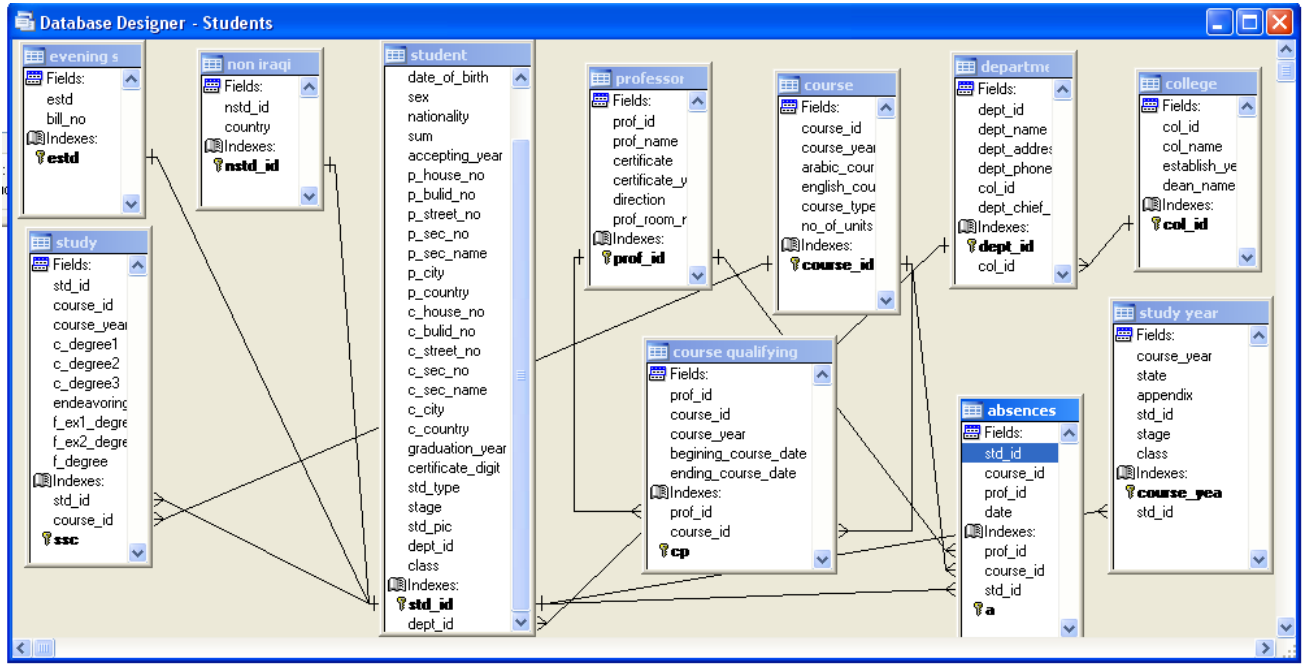

الثكل (9). قاعدة البيانات (Students) التي تضم الجداول

STUDENT, COURSE,PROFESSOR, DEPARTMENT,COLLEGE,EVENING STD, INTERNATIONAL STUDENT, STUDY YEAR,ABSENCE,COURSE QUALIFYING

تمت صياغة تكامل الإشارة (Referential Integrity) لقيمة للمفتاح الأساسي في الجداول التي يكون فيها مفتاحاً أجنبياً [4],[9] بحيث كانت قواعد التحديث منسابة(Cascade) وفيها يتم التحديث تلقائيا عند تغير الإنابر قيمة المفتاح الأساسي, أمـا قواعد الحذف فقد تم تجاهل حذف القيود المرتبطة بالمفتاح الأساسي تلافيا لضياع البيانات,اما عند الادخال فيجب ان تطابق القيمة المدخلة للمفتاح الأجنبي قيمة مفتاح أساسي , أو يرفض الإدخال,

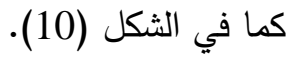




\begin{tabular}{|c|c|c|c|c|c|c|c|c|}
\hline Referential Inte & grity Builder & & & & & & & \\
\hline Rules for Updating & Rules for Dele & ting & Rule & $s$ for Inse & & & & \\
\hline Which rule do you & want to apply whe & en th & e key & alue in th & parent tal & is modified? & & \\
\hline (드ascade: upda & tes all related rec & ords & in the & child tabl & with the $\mathrm{n}$ & key value. & & \\
\hline Restrict: prohib & its the update if th & ere & are rel & ated reco & $\mathrm{sin}$ the $\mathrm{ct}$ & table. & & \\
\hline Ignore: allows $t$ & he update and le & aves & relate & records & the child & ble alone. & & \\
\hline Parent Table & Child Table & Upc & late & Delete & Insert & Parent Tag & Child Tag & 스 \\
\hline college & department & $\mathrm{Cas}$ & cade & Ignore & Restrict & col_id & col_id & \\
\hline course & absences & Cas & cade & Ignore & Restrict & course_id & course_id & \\
\hline course & study & & cade & lgnore & Restrict & course_id & course_id & \\
\hline course & course qualifying & Cas & cade & lgnore & Restrict & course_id & Course_id & \\
\hline department & student & Cas & cade & Ignore & Restrict & dept_id & dept_id & \\
\hline evening std & student & Cas & cade & Ignore & Restrict & estd & std_id & $v$ \\
\hline He elp & & & & & & $\underline{\mathrm{O} K}$ & & \\
\hline
\end{tabular}

الثكل (10). صياغة خصائص تكامل الإشارة بين المفتاح الأساسي والمفتاح الأجنبي في جداول قاعدة البيانات

فيما يلي سيتم ذكر عدد من(وليس كل) الواجهات الخاصدة بالتطبيق البرمجي وذلك لكثرتها, أو تشـابه قسم منها عند تنفيذ النظام وبعد عبور واجهة النظام الأولية والواجهة الأمنيـة يتم التوجه إلى واجهة الاختيارات كمـا في الشكل(11- أ) وعند اختيار إدخال أو تحديث البيانات يتم الذهاب إلى الثكل (11- ب) في واجهة جداول قاعدة

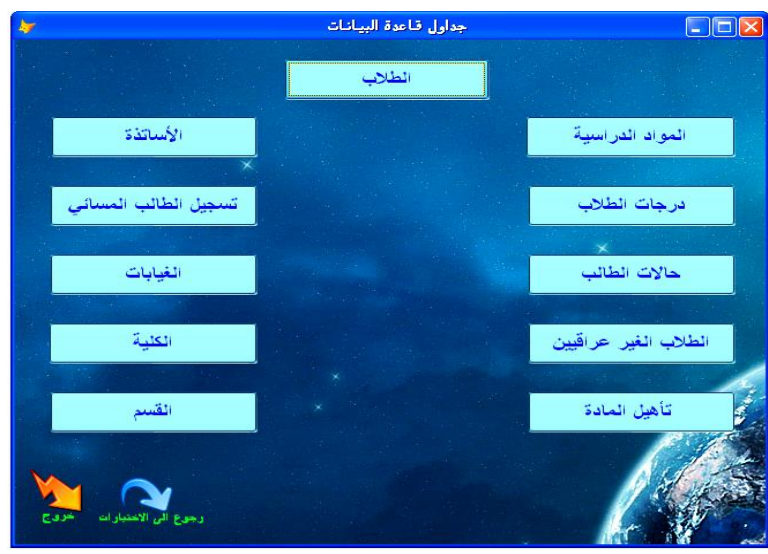

(ب)

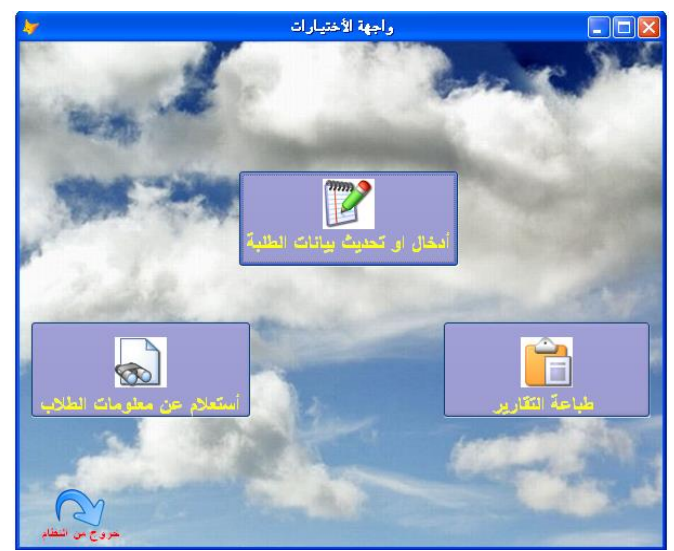

(I)

الثكل (13). واجهة الاختيارات وواجهة التعامل مع الجداول

عند اختيار درجات الطلبة من الشكل(11-ب) تظهر الواجهة كما في الثكل (12). 


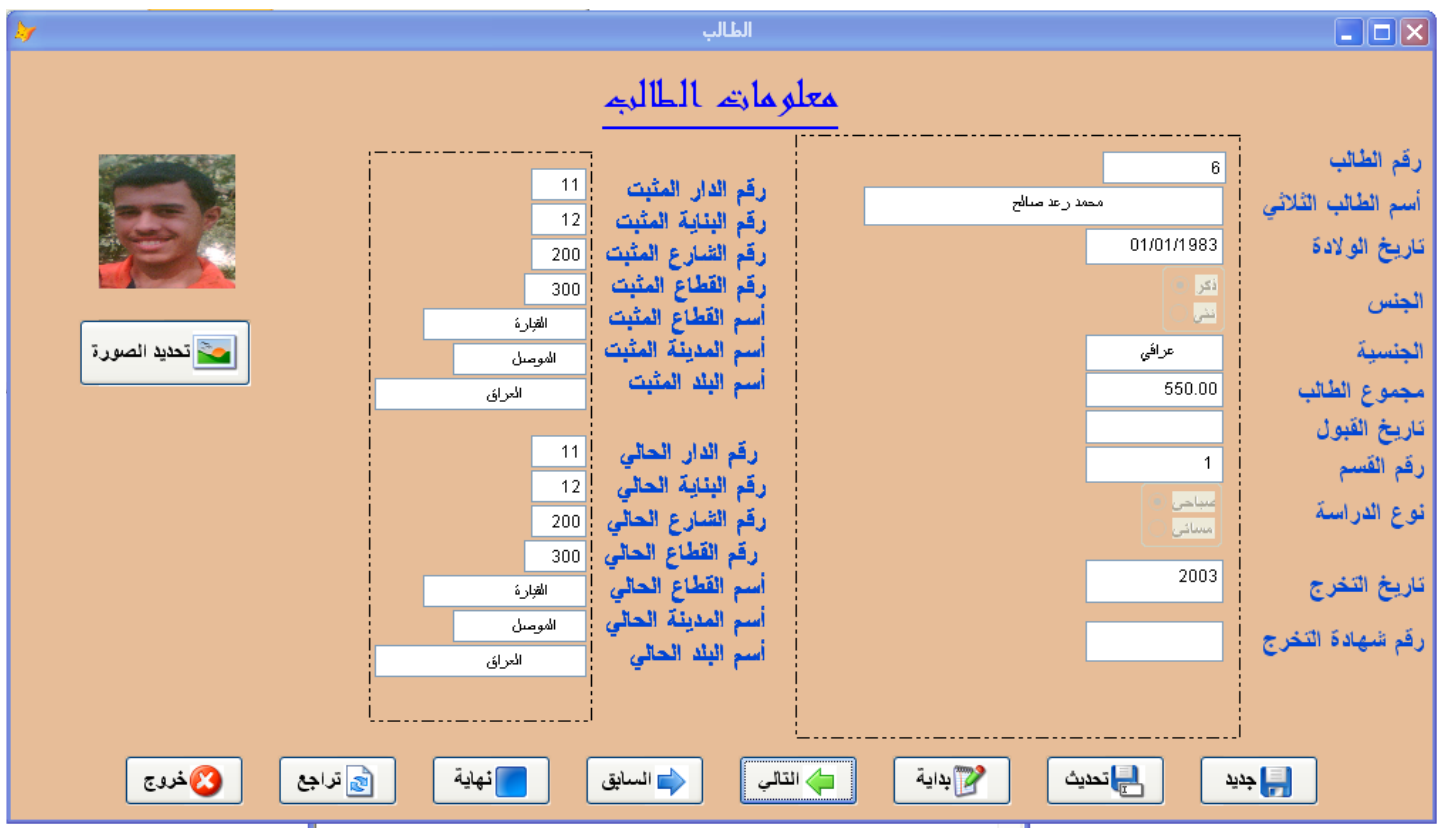

الثكل (12) واجهة معلومات الطالب

عند اختيار تأهيل المادة من الثكل (11-ب) تظهر الواجهة كما في الثكل (13).

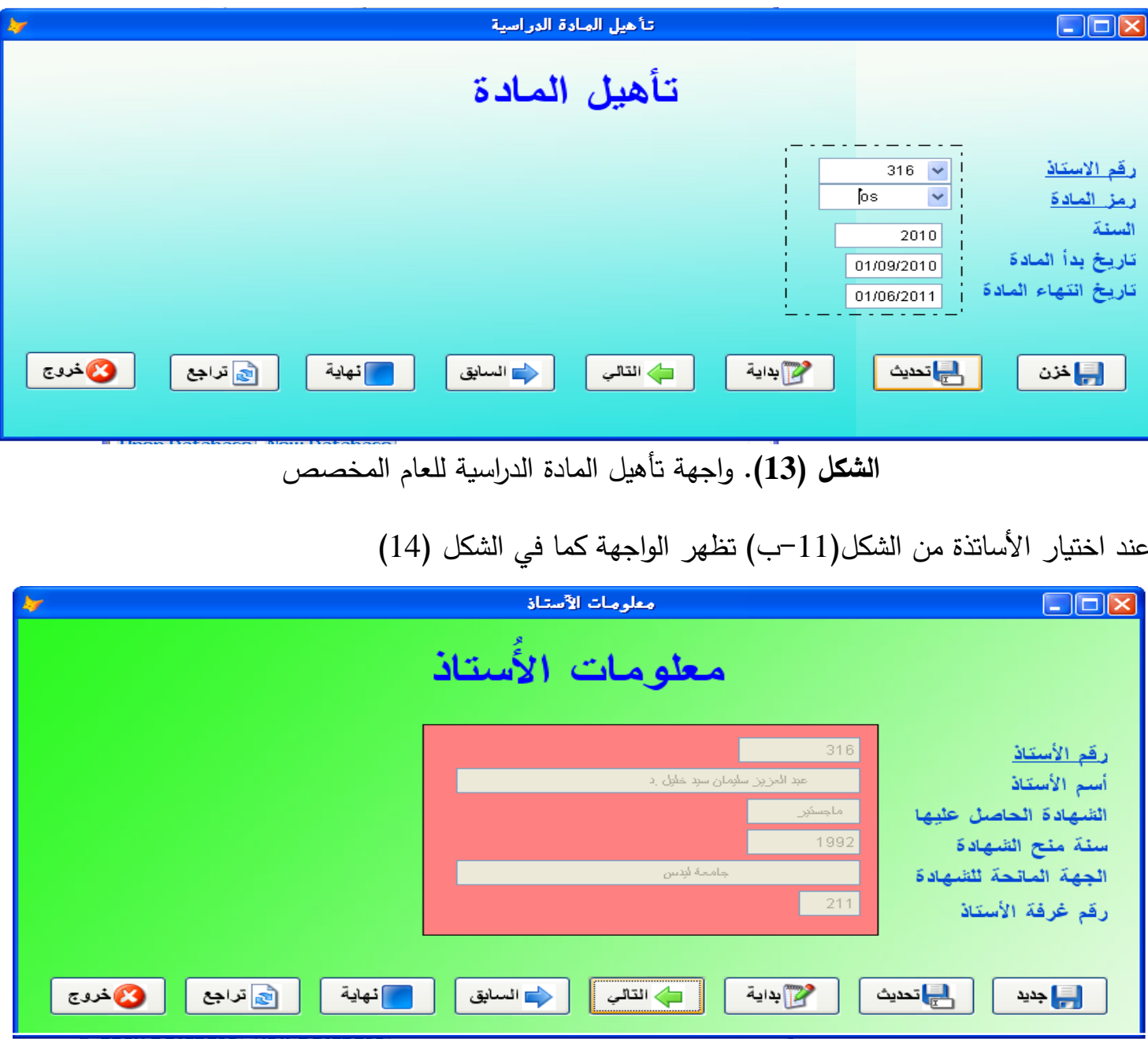

الشكل (14). واجهة معلومات الأستاذ 
عند اختيار الاستعلام عن معلومات طالب من الثكل(11-أ) تظهر الواجهة كما في الثكل (15).

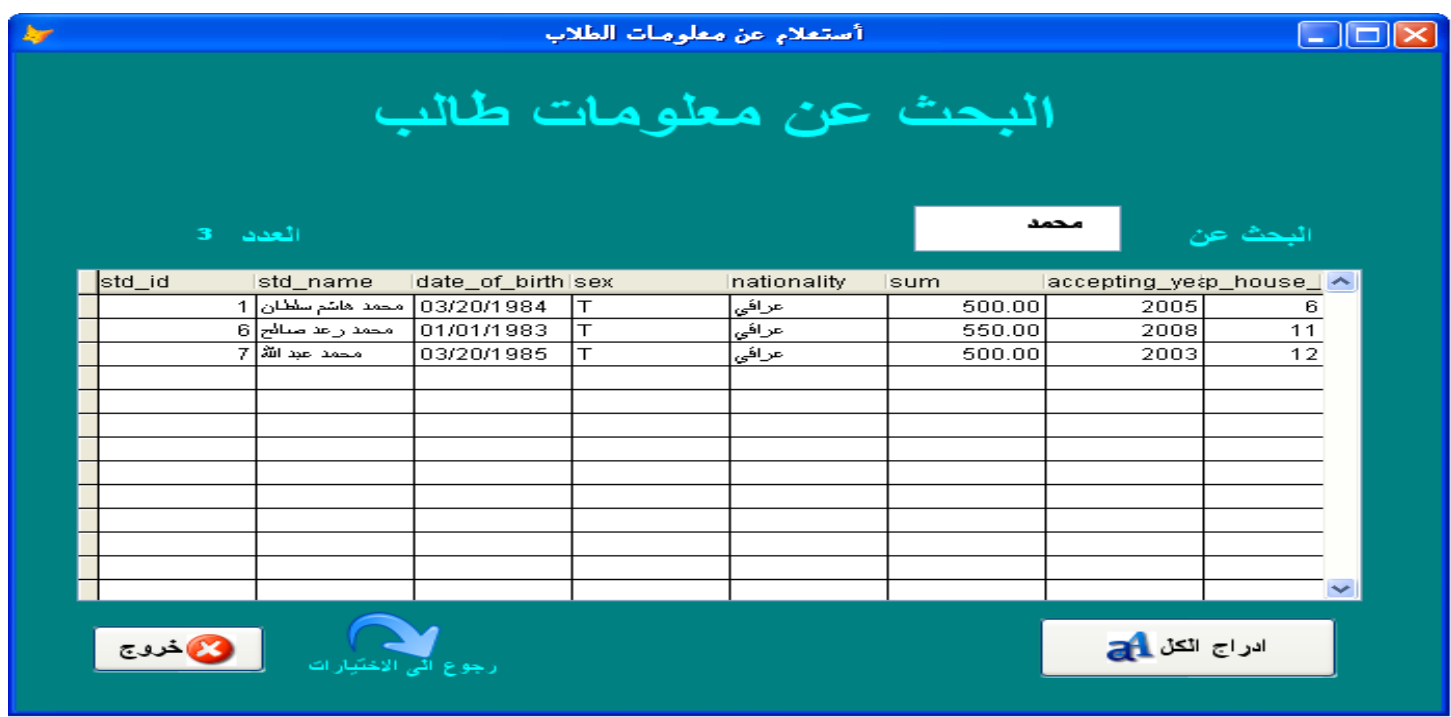

الثكل (15). واجهة البحث اليدوي المباشر باستخدام اسم الطالب

طباعة التقارير:

بالإمكـان طباعـة التقـارير مباشـرة إلـى الطابعـة المعرفـة على الحاسـبة, أو بالإمكـان عمـل مشــاهدة

قبل الطباعة, وهذه من الخصائص الجيدة في تطبيق Freview)

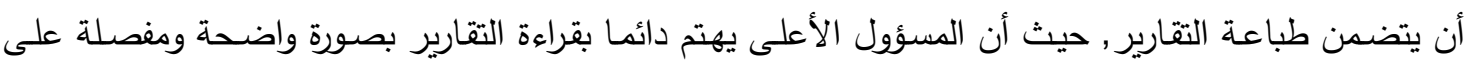
الورق. عند اختيار طباعة التقارير من الثكل(11-أ) تظهر الواجهة كما في الثكل (16). من خلا الواجهة في الثكل (16) يمكن طباعة التقارير عن أي اختيار حسب الحاجة, وان التقارير التي كونت في هذا البحث أساسية وليست مفصلة ,فقد تتطلب الحاجة خلال دورة حياة النظام أن تضاف تقارير أخرى أكثر تفصيلاً, عند اختيار "الطلاب" من الثكل (16) يكون بالإمكان معاينة التقرير الحاوي على البيانات ودوال إضافية بصيغة القيود (الأسطر ) كما في الثكل (17) أو بصيغة الجدول (الأعمدة).

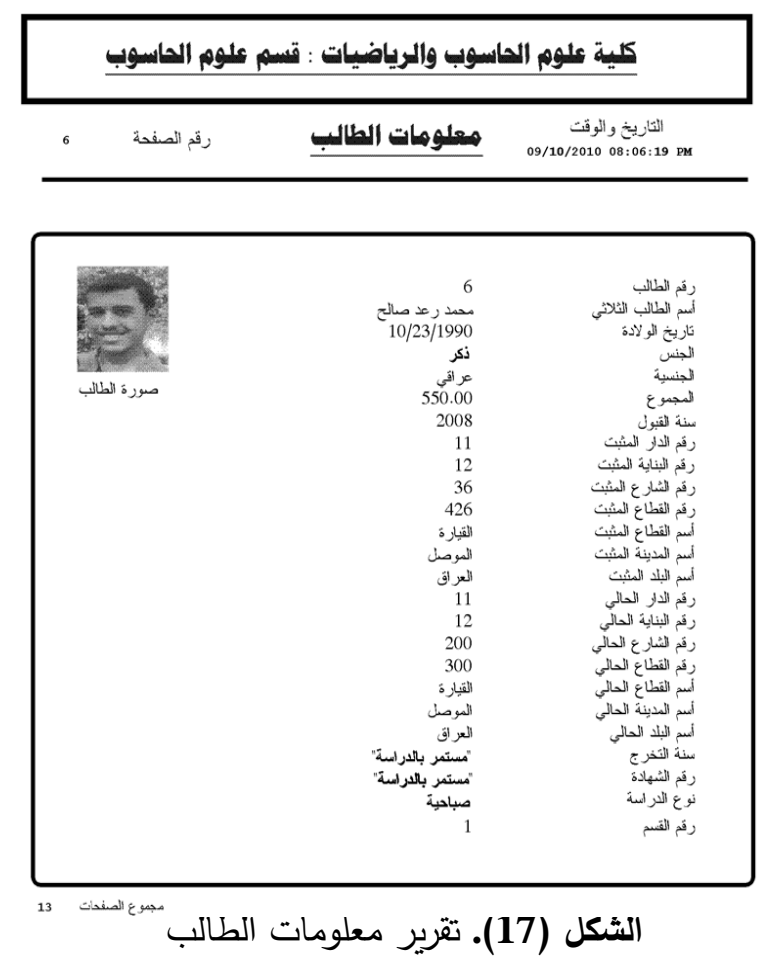

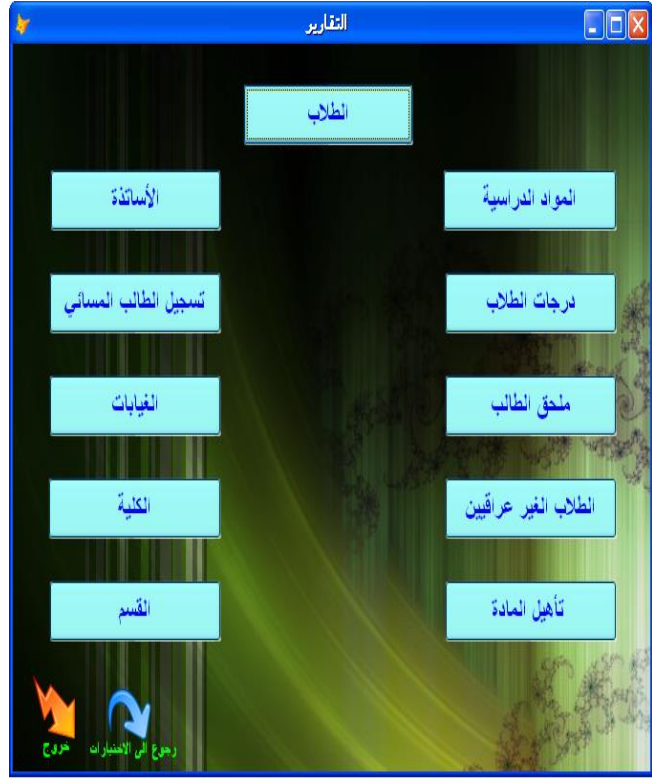

الثكل (16). واجهة التقارير 


$$
\text { 1. تمت الإحاطة بجميع ما متوفر ومطلوب من بيانات طلبة قسم علوم الحاسوب. }
$$

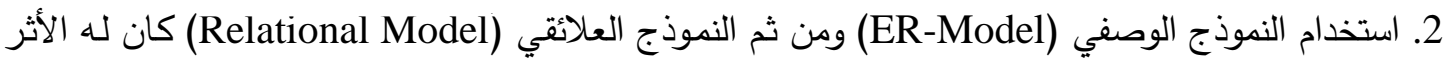

البالغ في تقوية قاعدة البيانات واكتشاف الأخطاء من البداية. 3. تم تطبيق خطوات التطبيع Normalization) للإيجاد أفضل الصيغ المعيارية Normal Forms للنموذج الفيات

العلائقي للتخلص من أية Anomalies. 4. اعتمادا على النموذج العلائقي فقد تم تكوين قاعدة بيانات متكاملة لطلبة قسم علوم الحاسوب حسب الجداول الوصفية لكل جدول.

5. في المرحلـة الأخيرة للتصديم الفعلي لـم تظهر الحاجـة إلى إجراء أيـة عمليـة Denormalization كون

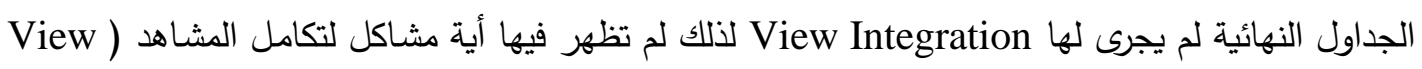

.(Integration Problems

6. زمن الوصول ومعالجة البيانات من حيث الإدخال والاسترجاع والتحديث والحذف لا يكاد يذكر.

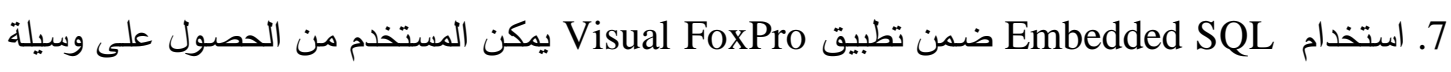
جيدة لاسترجاع البيانات, وكذلك اعتمدت عليه بعض التقارير • 8. وجود الرمز الموحد للطالب يساعد بفعالية جيدة في أية عملية بحث أو استقصاء أو طباعة أي تقرير . الاستنتاجات :

1. إن التحليل الجيد للنظام من أول المراحل وبأدق التفاصيل يجعل أساس قاعدة البيانات قوياً لأنه بدون التحليل

$$
\text { للنظام لا يمكن تكوين ترابط جيد ما بين محتويات النظام. }
$$

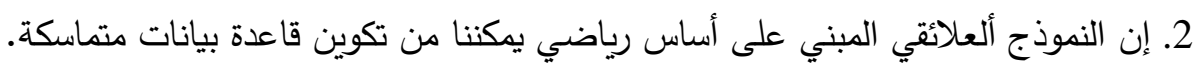

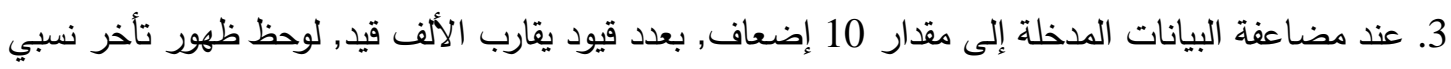
ضئيل عند معالجة البيانات بمقدار عدد من الثواني وحسب موقع القيد. 4. لا يؤثر تسلسل خزن الصفات في الجدول على محتوى الجدول, بالإمكان خزن الصفات بتسلسل عشوائي في الجدول. 5. الترحيل الآلي لبيانات الطلبة يمر بسلاسة عند إجراءه في نهاية كل عام دراسي.

1. تكوين قاعدة بيانات موزعة لكي يتم تطبيق النظام على طلبة الكلية بأقسامها الأربعة. 2. إضافة خاصية البرمجة باستخدام Client - Server الموجودة في اللغة البرمجية لدعم النقطة 1 أعلاه لتمكين الوحدات الإدارية ذات العلاقة ببيانات الطلبة من تحقيق الاستفادة منها. 3. إعادة صياغة التقارير بصورة تفصيلية أكثر من خلال الحاجات الجديدة للقسم. 4. إضافة إمكانية طباعة الهويات الخاصة بالطلبة كون جميع البيانات متوفرة. 5. إضافة إمكانية طباعة النتائج للامتحانات مع الأخذ بنظر الاعتبار النقاط 2-5 أعلاه. 
6. محاولة عمل ترابط مابين النظام ونظم إدارة قواعد البيانات لتدريسيي الكلية ضمن بيانات موظفي الكلية. 7. الارتقاء بالنظام إلى مستوى مستودعات البيانات إذا تم تطبيقه على بيانات طلبة جامعة الموصل وبالتوافق

مع النقطتين 1 و 2 أعلاه.

8. إجراء التحديث المستمر على النظام لتفادي الفشل أو الانهيار. 


\section{|"مصادر}

[1] Silberschatz, Korth and Sudarshan 2010, "Database System Concepts", 6 ${ }^{\text {th }}$ Edition, McGraw-Hill.

[2] Jeffrey A. Hoffer, Joy F. George, Joseph S. Valacich 2005, "Modern Systems Analysis and Design" $4^{\text {th }}$ Edition, Pearson Printice Hall.

[3] Date, C.J. 2000, "The Database Relational Model", Addison-Wesley.

[4] Fred R. McFadden 1999, "Modern Database Management Systems", 5 Edition, Addison -Wesly.

[5] Codd, E. F. 1993, "Beyond decision support, in Computer world", Codd, S. B., Salley, C. T.

[6] C. J. Date, 1990, "An Introduction to Database Systems", Volume I, 5 Edition, Addison-Wesley.

[7] Claudia Imhoff, Nicholas Galemmo, and Jonathan G. Geiger, 2003, "Mastering Data warehouse Design, Relational And Dimensional Techniques", Wiley Publishing, Inc.

[8] J.M. Smith, D.C.P. Smith 1977, "Database Abstractions: Aggregation and Generalization", ACM TODS 2, pp(105-133).

[9] Codd, E.F 1970, "A Relational Model of Data for Large Shared Data Banks", Communications of the ACM 13, Vol. 13 No. 6 pp(377-387). 